\title{
Advanced spectrophotometric chemometric methods for resolving the binary mixture of doxylamine succinate and pyridoxine hydrochloride
}

\author{
PLAMEN KATSAROV ${ }^{1,6^{*}}$ \\ GEORGI GERGOV ${ }^{2}$ \\ AYLIN ALIN ${ }^{3}$ \\ BISSERA PILICHEVA ${ }^{1,6}$ \\ YAHYA AL-DEGS ${ }^{4}$ \\ VASIL SIMEONOV \\ MARGARITA KASSAROVA ${ }^{1,6}$ \\ ${ }^{1}$ Department of Pharmaceutical Sciences \\ Faculty of Pharmacy, Medical \\ University-Plovdiv, 4002 Plovdiv, Bulgaria \\ ${ }^{2}$ Department of Chemistry, Faculty \\ of Pharmacy, Medical University Sofia \\ 1000 Sofia, Bulgaria \\ ${ }^{3}$ Department of Statistics, Dokuz Eylul \\ University, 35160 Izmir, Turkey \\ ${ }^{4}$ Chemistry Department, The Hashemite \\ University, P. O. Box 150459 Zarqa 13115 \\ Jordan \\ ${ }^{5}$ Laboratory of Chemometrics and \\ Environmetrics, Faculty of Chemistry \\ and Pharmacy, Sofia University \\ "St. Kliment Ohridski", 1164 Sofia, Bulgaria \\ ${ }^{6}$ High-technological Center of Emergency \\ Medicine, Plovdiv, Bulgaria
}

Accepted November 15, 2017

Published online December 8, 2017

\begin{abstract}
The prediction power of partial least squares (PLS) and multivariate curve resolution-alternating least squares (MCR-ALS) methods have been studied for simultaneous quantitative analysis of the binary drug combination - doxylamine succinate and pyridoxine hydrochloride. Analysis of first-order UV overlapped spectra was performed using different PLS models - classical PLS1 and PLS2 as well as partial robust M-regression (PRM). These linear models were compared to MCR-ALS with equality and correlation constraints (MCR-ALS-CC). All techniques operated within the full spectral region and extracted maximum information for the drugs analysed. The developed chemometric methods were validated on external sample sets and were applied to the analyses of pharmaceutical formulations. The obtained statistical parameters were satisfactory for calibration and validation sets. All developed methods can be successfully applied for simultaneous spectrophotometric determination of doxylamine and pyridoxine both in laboratory-prepared mixtures and commercial dosage forms.
\end{abstract}

Keywords: doxylamine succinate, pyridoxine hydrochloride, binary mixture, partial least squares (PLS), partial robust M-regression (PRM), multivariate curve resolution-alternative least squares (MCR-ALS)

Analytical separation techniques for simultaneous determination of drugs have gained popularity in the last few years due to the intensive development of fixed-dose combination drug products (FDCs). One of the latest FDCs on the pharmaceutical market, approved in 2013 by the US Food and Drug Administration (FDA), is the combination of doxylamine succinate and pyridoxine hydrochloride (1).

\footnotetext{
*Correspondence; e-mail: plamen.katsarov@yahoo.com
} 


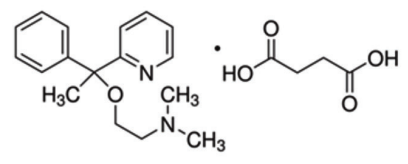

Doxylamine succinate

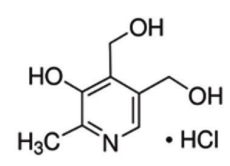

Pyridoxine hydrochloride

Fig. 1. Chemical structures of doxylamine succinate (DOX) and pyridoxine hydrochloride (PYR).

Doxylamine succinate (DOX) (Fig. 1) is a first-generation antihistaminic with antimuscarinic action and sedative effect. Pyridoxine hydrochloride (PYR) is a water soluble vitamin involved in different biochemical reactions in the body. The DOX and PYR combination has recently gained great interest because it is currently one of the few medicines on the pharmaceutical market that have been clinically proven to be effective and safe for the treatment of nausea and vomiting during pregnancy (2).

Nowadays, spectrophotometric analytical methods combined with powerful signal processing algorithms have been applied for quantitative determination of drugs in many pharmaceutical formulations. Partial least squares (PLS1, PLS2) (3) and advanced partial robust M-regression (PRM), which do not require outlier detection (4), have attracted the attention of pharmaceutical analysts owing to their superb performance in multicomponent analysis. Multivariate curve resolution-alternating least squares with a correlation constraint (MCR-ALS-CC) is another chemometric technique that has enabled successful quantification of spectrally-overlapping drugs $(5,6)$. Common PLS models require not only standards but also possible interferences in a dosage form to be known. However, this is not necessary when using MCR-ALS, which has the advantage of analysing pharmaceutical formulations with unknown excipients (7).

Official pharmacopeial methods are available for the determination of DOX and PYR individually (8), but only few analytical methods have been developed for simultaneous evaluation of the drugs in common dosage forms. Separation-based methods such as reversed-phase high-performance liquid chromatography (RP-HPLC) and high-performance thin-layer chromatography (HPTLC) have been reported for DOX and PYR quantification $(9,10)$. Graphical derivative spectrophotometric techniques, including derivative ratio zero-crossing and double divisor derivative ratio spectra methods, have been applied for ternary mixtures containing DOX and PYR (11). Spectrophotometric determination of the two drugs at acidic $\mathrm{pH}$ has been reported (12). However, at neutral $\mathrm{pH}$, intense spectral overlapping of these drugs is observed, which makes their simultaneous determination more complicated. In such media, only the first-derivative zero-crossing method has been used with limited applicability for the current system (13). Multivariate calibration techniques - classical least squares (CLS), inverse least squares (ILS), principal components regression (PCR) and PLS - have been applied for analysing only ternary mixtures containing DOX and PYR in combination with folic acid (14).

Quantitative analysis of DOX is additionally hindered due to the intense overlapping of its spectrum between 240 and $280 \mathrm{~nm}$, with the signal of the tautomeric form of PYR predominant at neutral $\mathrm{pH}$. In such a case, the application of CLS and ILS may be limited. However, other advanced multivariate techniques such as PLS, PRM and MCR-ALS should give better results. In fact, both CLS and ILS are not factor-based methods and hence intense spectral overlapping and high collinearity in spectral data would retard the applica- 
tion of such models. However, PCR, PLS and MCR-ALS methods are factor-based models and hence they can handle spectral overlapping and high collinearity by adjusting a number of factors to end up with accurate prediction of both drugs in solution.

The aim of the current work is to assess the performance of some advanced chemometric techniques to eliminate the intense spectral overlapping between DOX and PYR at neutral $\mathrm{pH}$ prior to their quantification. To the best of our knowledge, this is the first study applying PLS1, PLS2, PRM and MCR-ALS for simultaneous spectrophotometric determination of DOX and PYR, both in artificial mixtures and in pharmaceutical dosage forms. The developed models can be used in the pharmaceutical practice to analyse this drug combination in nasal, pulmonary or buccal formulations, which release the incorporated drugs at neutral $\mathrm{pH}$.

\section{EXPERIMENTAL}

\section{Apparatus}

A UV-visible spectrophotometer Evolution 300 (Thermo Fisher Scientific, USA) was used to record the absorption spectra of all solutions. Scanning was carried out in the range of $200-400 \mathrm{~nm}$ at $1.0-\mathrm{nm}$ intervals with $1.00-\mathrm{cm}$ quartz cells. VISIONpro ${ }^{\mathrm{TM}}$ software (Thermo Fisher Scientific) was utilized for data acquisition.

\section{Materials}

Doxylamine succinate and pyridoxine hydrochloride were purchased from Sigma Aldrich, USA. Commercial dosage forms - hard modified-release capsules, each containing $10 \mathrm{mg}$ of DOX and $10 \mathrm{mg}$ of PYR - were purchased from the pharmaceutical market under the brand name Cariban ${ }^{\circledR}$ (Laboratorios Inibsa, S.A., Spain). Phosphate-buffered saline (PBS) of pH 6.8 was freshly prepared according to the European Pharmacopoeia (8) and was used as solvent for all the prepared stock and working solutions.

\section{Preparation of DOX and PYR standard solutions}

Accurately weighed $20 \mathrm{mg}$ of DOX was transferred into a volumetric flask of $200 \mathrm{~mL}$ and dissolved in PBS, $\mathrm{pH}$ 6.8. The solution was sonicated for $15 \mathrm{~min}$ at room temperature and the content was completed to the mark with PBS. In this way, a stock solution of DOX with a concentration of $100 \mu \mathrm{g} \mathrm{mL}^{-1}$ was prepared. The same procedure was applied to obtain a stock solution of PYR with a concentration of $100 \mu \mathrm{g} \mathrm{mL}^{-1}$. Stock solutions of DOX and PYR were stored at $25 \pm 0.5^{\circ} \mathrm{C}$ until analysis. All working solutions were prepared from the stock solutions after appropriate dilutions.

\section{Preparation of dosage form solutions}

Twenty Cariban ${ }^{\circledR}$ capsules were emptied and their content was weighed. Capsule contents were mixed in a mortar and ground to a fine powder. An accurately weighed amount of the mixed powder (equivalent to $10 \mathrm{mg}$ of DOX and $10 \mathrm{mg}$ of PYR) was transferred into $150 \mathrm{~mL}$ of PBS (pH 6.8), sonicated for $15 \mathrm{~min}$ and then made up to $200 \mathrm{~mL}$ with PBS in a 
P. Katsarov et al:: E Advanced spectrophotometric chemometric methods for resolving the binary mixture of doxylamine succinate and pyridoxine hydrochloride, Acta Pharm. 68 (2018) 61-73.

Table I. Experimental design of the calibration and validation set mixtures

\begin{tabular}{cccccc}
\hline & Calibration set $^{\mathrm{a}}$ & & \multicolumn{3}{c}{ Validation set $^{\mathrm{a}}$} \\
\hline Mixture & DOX & PYR & Mixture & DOX & PYR \\
\hline 1 & 30.0 & 15.0 & 1 & 30.0 & 10.0 \\
2 & 30.0 & 25.0 & 2 & 40.0 & 10.0 \\
3 & 80.0 & 15.0 & 3 & 30.0 & 15.0 \\
4 & 80.0 & 25.0 & 4 & 30.0 & 20.0 \\
5 & 20.0 & 20.0 & 5 & 30.0 & 30.0 \\
6 & 90.0 & 20.0 & 6 & 40.0 & 15.0 \\
7 & 55.0 & 13.0 & 7 & 40.0 & 20.0 \\
8 & 55.0 & 27.0 & 8 & 50.0 & 10.0 \\
9 & 55.0 & 20.0 & & & \\
10 & 55.0 & 20.0 & & & \\
11 & 55.0 & 20.0 & & & \\
\hline
\end{tabular}

${ }^{\mathrm{a}}$ Concentrations of mixture components in $\mu \mathrm{g} \mathrm{mL}^{-1}$.

volumetric flask. A stock solution with a concentration of $50 \mu \mathrm{g} \mathrm{mL}^{-1}$ for DOX and $50 \mu \mathrm{g} \mathrm{mL}$ for PYR was thus prepared and filtered using a $0.45 \mu \mathrm{m}$ syringe filter. After appropriate dilution, a working solution with a concentration of $30 \mu \mathrm{g} \mathrm{mL} \mathrm{L}^{-1}$ for DOX and $30 \mu \mathrm{g} \mathrm{mL}^{-1}$ for PYR was prepared and scanned over the spectral range between 230 and $400 \mathrm{~nm}$.

\section{Experimental design}

A calibration set of mixtures was constructed with the aid of Chemoface statistical software using a central composite design (CCD), as shown in Table I (15). It comprised 11 mixtures containing different concentrations of DOX and PYR in the concentration range of 30-90 $\mu \mathrm{g} \mathrm{mL}-1$ for DOX and 10-30 $\mu \mathrm{g} \mathrm{mL}^{-1}$ for PYR. Another 8 mixtures were used as a validation set to test the predictive power of the developed chemometric models.

\section{Multivariate calibration techniques}

The analytical performance of classical PLS models (PLS1 and PLS2) (16) was compared to the linear PRM model (17). PLS is an inverse calibration procedure for developing a quantitative relationship between several predictor variables $X$ (spectra) and dependent variables $C$ (concentration) according to the equation:

$$
C_{(n \times s)}=X_{(n \times k)} \times B_{(k \times s)}
$$

where $C$ is the matrix sized $n \times s, n$ is the number of samples, $s$ is the number of components, $X$ is the $n \times k$ centred data matrix containing the absorbances of standard solutions measured at $k$ wavelengths, and $B$ is the $k \times s$ regression coefficient matrix. When only one 
drug component is considered ( $s=1)$, the PLS method is called PLS1. For $s>1$, it is known as PLS2.

MCR-ALS optimization algorithm was also tested (18-21). MCR is based on a bilinear additive model, which can be expressed by the following equation:

$$
X=C \times S^{T}+E
$$

where $X$ is the spectral data matrix of $(n+m) \times k, C$ is the concentration profile of all components and $S^{T}$ is the matrix of the corresponding pure spectra. Matrix $E$ contains the unmodelled part of the data, which is not explained by the bilinear model.

Information about singular value decomposition (SVD), non-negativity, equality and correlation constraints is given in the literature $(5,19-22)$.

Assessment of multivariate calibration methods for drug quantification. - Root mean square errors of calibration (RMSEC), root mean square errors of prediction (RMSEP), relative error of prediction $(R E)$ and squared correlation coefficients $\left(R^{2}\right)$ were estimated using the equations:

$$
\begin{gathered}
R M S E P=\sqrt{\frac{\sum_{i=1}^{m}\left(C_{\mathrm{pred}, i}-C_{\text {true }, i}\right)^{2}}{m}} \\
R E(\%)=100 \times \sqrt{\frac{\sum_{i=1}^{m}\left(C_{\mathrm{pred}, i}-C_{\text {true }, i}\right)}{\sum_{i=1}^{m} C_{\mathrm{pred}, i}^{2}}}
\end{gathered}
$$

where $C_{\text {pred }, i}$ and $C_{\text {true }, i}$ are predicted and true concentrations of each component in sample $i$, resp., and $\hat{C}_{\text {true }}$ is the average true concentration. $n$ and $m$ are the number of samples in calibration and prediction sets, resp. RMSEC characterizes the quality of calibration models, while RMSEP and $R E$ values not only assess the quality of prediction but also help select the optimal number of latent variables (LVs). The squared correlation coefficient $\left(R^{2}\right)$, measuring how much variance of concentration is explained by the absorbance spectra values, characterizes model quality and helps compare the developed methods.

Lack of fit $(L O F)$ values and percent of variance were used for assessment of the MCRALS method. These statistical parameters were calculated as follows:

$$
\begin{gathered}
\operatorname{LOF}(\%)=100 \times \sqrt{\frac{\sum_{i j}\left(d_{i j}-d_{i j}^{*}\right)^{2}}{\sum_{i j} d_{i j}^{2}}} \\
\text { Variance }(\%)=100 \times \frac{\sum_{i j} d_{i j}^{2^{*}}}{\sum_{i j} d_{i j}^{2}}
\end{gathered}
$$

where $d_{i j}$ and $d_{i j}^{*}$ are the experimental and MCR-ALS-predicted absorbance values, resp. 


\section{Chemometric software}

All spectra were converted to digital values and exported to MatLab for subsequent calculation. Numerical calculations were made using MatLab ${ }^{\circledR}$ 2013a (Mathworks, Natick, MA, USA). PLS2 calibration was carried out using the MVC1 ${ }^{\circledR}$ MatLab toolbox (23), while the TOMCAT ${ }^{\circledR}$ MatLab toolbox (24) was used to run PLS1 and RPM. MCR-ALS routines were performed using multivariate curve resolution-alternating least squares subroutines (25). All programs were available on the internet.

\section{RESULTS AND DISCUSSION}

Series of spectral tests were carried out to evaluate the influence of $\mathrm{pH}$ on the spectral behaviour of DOX and PYR. Due to tautomeric equilibrium, the UV-spectrum of PYR was strongly dependent on $\mathrm{pH}$ (26). At acidic $\mathrm{pH}, \mathrm{N}$-protonation of PYR took place and an absorption maximum was observed at $290 \mathrm{~nm}$. However, at neutral $\mathrm{pH}$, the zwitterionic form of PYR prevailed and, besides the absorption maximum at $324 \mathrm{~nm}$, a new maximum appeared at $254 \mathrm{~nm}$. The UV spectra of DOX and PYR at pH 6.8 are represented in Fig. 2.

At neutral $\mathrm{pH}$ (6.8), the drugs exhibited intense spectral overlapping over the range between 200 and $400 \mathrm{~nm}$. DOX showed one suitable wavelength for quantitative analysis at $260 \mathrm{~nm}$, while PYR could be quantified at two wavelengths: 254 and $324 \mathrm{~nm}$. As shown in Fig. 2, in the region between 240 and $280 \mathrm{~nm}$ there was an intense overlap between the spectra of the two drugs, which hindered direct determination of DOX. For the current system, a high degree (67\%) of spectral overlap between DOX and PYR was estimated (27).

Besides the spectral overlap between the two drugs, a non-linearity test was also carried out to verify the relationship between drug content and PLS-scores, as outlined in the literature (28). The results showed a linear relationship between both drugs and PLSscores indicating applicability of the linear models (PLS1, PLS2 and PRM) for handling the current analytical system.

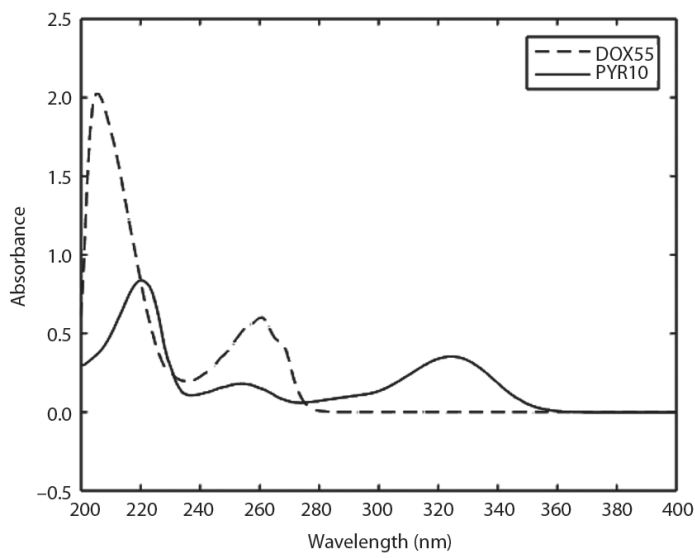

Fig. 2. Absorption spectra of DOX $\left(55 \mu \mathrm{g} \mathrm{mL}^{-1}\right)$ and PYR $\left(10 \mu \mathrm{g} \mathrm{mL} \mathrm{L}^{-1}\right)$ at $\mathrm{pH} 6.8$. 


\section{PLS models}

Three PLS versions (PLS1, PLS2 and PRM) were applied to calibrate the drugs in the solution. Wavelength selection was carried out before running PLS calibration. The spectral region from 200 to $230 \mathrm{~nm}$ was removed due to strong solvent interference. The remaining spectral range $230-400 \mathrm{~nm}$ (171 spectral data) was included in numerical analysis to calibrate DOX and PYR. The two components had no spectral absorbance at further wavelengths. For the PLS methods, the spectral data were placed in three different matrices: the main calibration matrix $X(11 \times 171)$, a validation matrix $(8 \times 171)$, designed as shown in Table I, and a dosage form matrix $(5 \times 171)$. Mean centring was chosen as a preprocessing step in PLS1 and PLS2, while median centring was chosen as a preprocessing step in PRM. With PLS1 regression, a model was built for each drug using its concentration vector, whereas with PLS2 regression, concentrations of both drugs were used simultaneously. The prediction ability of the PLS methods was not satisfactory, which could be attributed to the intense overlapping and the possible existence of outliers. The later drawback was eliminated by constructing a robust version of PLS (i.e., PRM), which automatically removed outliers before creating the model.

The optimum number of PLS variables was determined by the minimum values of RMSEP and RE. Selection of the correct number of components was important to avoid

Table II. Calibration and validation parameters and figures of merits for DOX and PYR for PLS1, PLS2 and RPM methods

\begin{tabular}{lcccccc}
\hline \multirow{2}{*}{ Parameter } & \multicolumn{2}{c}{ PLS1 } & \multicolumn{2}{c}{ PLS2 } & \multicolumn{2}{c}{ RPM } \\
\cline { 2 - 7 } & DOX & PYR & DOX & PYR & DOX & PYR \\
\hline Calibration & & & & & & \\
\hline PCs & 3 & 4 & 4 & 4 & 3 & 4 \\
$R M S E C\left(\mu \mathrm{gL}^{-1}\right)$ & 0.3701 & 0.0879 & 0.3582 & 0.0835 & 0.2987 & 0.0648 \\
$R E(\%)$ & 0.6203 & 0.4283 & 0.6002 & 0.4067 & 0.8084 & 0.4226 \\
$R^{2}$ & 0.9999 & 0.9998 & 0.9999 & 0.9998 & 0.9998 & 0.9998 \\
\hline Validation & & & & & & \\
\hline$R M S E P\left(\mu \mathrm{g} \mathrm{mL} \mathrm{L}^{-1}\right)$ & 0.4220 & 0.0886 & 0.3487 & 0.0943 & 0.4200 & 0.0875 \\
$R E(\%)$ & 1.1432 & 0.5063 & 0.9446 & 0.5388 & 1.1379 & 0.4998 \\
$R^{2}$ & 0.9991 & 0.9999 & 0.9994 & 0.9999 & 0.9987 & 0.9999 \\
Figures of merit & & & & & & \\
Sensitivity & 0.0382 & 0.0494 & 0.0351 & 0.0472 & 0.0408 & 0.0450 \\
LOD $^{\mathrm{b}}$ & $0.61-0.89$ & $0.25-0.30$ & $0.59-0.91$ & $0.24-0.29$ & $0.60-0.88$ & $0.23-0.29$ \\
LOQ $^{\mathrm{c}}$ & $1.83-2.68$ & $0.75-0.91$ & $1.77-2.73$ & $0.72-0.87$ & $1.81-2.59$ & $0.73-0.89$ \\
\hline
\end{tabular}

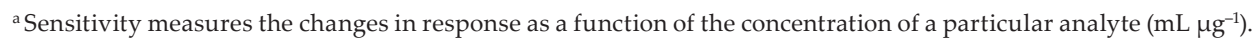

${ }^{\mathrm{b}}$ Limit of detection is the lowest concentration of an analyte that can be detected, but not necessarily quantified $\left(\mu \mathrm{g} \mathrm{mL} \mathrm{L}^{-1}\right)$.

${ }^{c}$ Limit of quantification is the lowest concentration of an analyte that can be quantitatively determined with suitable precision and accuracy $\left(\mu \mathrm{g} \mathrm{mL} \mathrm{m}^{-1}\right)$. 
model overfitting. As shown in Table II, four latent variables were chosen for both drugs in the PLS2 model. For PLS1 and PRM, the optimum number of variables was different: three for DOX and four for PYR. As the optimum number of latent variables (3 to 4 ) was higher than the number of components (2 solutes), a serious rank-deficiency in matrices was encountered. This was probably caused by the intense spectral overlap. In that case, PLS and PRM regression seemed to be the best choice for modelling such data with a multicollinearity problem.

The eight synthetic mixtures used to validate calibration models were analysed with PLS1, PLS2 and PRM for each drug. The "predicted vs. true" plots were constructed to check out the performance of the models. The final statistical parameters are presented in Table II. The parameters: $R M S E C, R M S E P, R E$ and $R^{2}$ provided the basis for comparison between the models.

DOX was predicted by the PLS calibration with RMSEC $0.2987-0.3701 \mu \mathrm{g} \mathrm{mL}^{-1}$, while a better prediction of PYR was observed with RMSEC $0.0648-0.0879 \mu \mathrm{g} \mathrm{mL}^{-1}$. At the same time, $R E$ values also indicated better prediction of PYR $(0.4067-0.4283 \%)$ compared to DOX $(0.6002-0.8084 \%)$. The validation statistical parameters for both components have similar values for the three PLS models.

In general, statistical parameters for all PLS models of DOX were worse compared to PYR. The possible reason was the lack of a selective region in the spectrum of DOX due to the intense overlapping with the spectrum of PYR (Fig. 2).

Figures of merit (FOM) - sensitivity, limit of detection $(L O D)$ and limit of quantification $(L O Q)$ - were also calculated for the PLS models. LOD and LOQ intervals were provided instead of single values (29). PYR turned out to have higher sensitivity with lower values of $L O D$ and $L O Q$ compared to DOX. This could be attributed to the vast region of the PYR spectrum in which it was not overlapped by the DOX spectrum.

\section{MCR-ALS method}

For the MCR-ALS method, the calibration matrix $(11 \times 171)$ and the validation matrix $(8 \times 171)$ were disposed in two different column-wise augmented matrices, which were used for calibration $(22 \times 171)$ and validation $(19 \times 171)$. For dosage forms, the calibration matrix $(11 \times 171)$ and the dosage form matrix $(5 \times 171)$ were disposed in a column-wise augmented matrix $(16 \times 171)$.

SVD was used to decompose the calibration data matrix and two singular values were visually inspected. It was assumed that there were two significant factors and no interferences present in the dosage form samples. Further, the pure spectral profiles of the standard compounds, DOX and PYR, were used as initial estimates to check the MCR-ALS solutions and also to reduce the effects of rotational ambiguities. To test the ability of MCR-ALS for internal validation, the calibration data set was used as a second subset to perform model validation.

Non-negativity constraint was used in order to ensure that the concentrations and the spectra of the components had positive values. Two other constraints were applied during the ALS optimization procedure. Initially, the MCR-ALS model was tested with known pure spectral profiles (equality constraint). Afterwards, the correlation constraint was used to eliminate the dosage form matrix effects. The correlation constraint gave better spectral profiles than the equality one. The profiles of both drugs generated by MCR-ALS- 
P. Katsarov et al.: E Advanced spectrophotometric chemometric methods for resolving the binary mixture of doxylamine succinate and pyridoxine hydrochloride, Acta Pharm. 68 (2018) 61-73.

Table III. Calibration and validation parameters for DOX and PYR for MCR-ALS and MCR-ALS-CC methods

\begin{tabular}{|c|c|c|c|c|}
\hline \multirow{2}{*}{ Parameter } & \multicolumn{2}{|c|}{ MCR-ALS } & \multicolumn{2}{|c|}{ MCR-ALS-CC } \\
\hline & DOX & PYR & DOX & PYR \\
\hline \multicolumn{5}{|l|}{ Calibration } \\
\hline PCs & 2 & 2 & 2 & 2 \\
\hline Number of iterations & 15 & 12 & 4 & 3 \\
\hline LOF (\%) (PCA) & 0.3712 & 0.5357 & 0.3715 & 0.5497 \\
\hline LOF (\%) (exp.) & 0.7855 & 0.8753 & 0.7856 & 0.8840 \\
\hline Variance (\%) & 99.9938 & 99.9923 & 99.9938 & 99.9922 \\
\hline RMSEC $\left(\mu \mathrm{g} \mathrm{mL} L^{-1}\right)$ & 0.6117 & 0.2117 & 0.6106 & 0.2088 \\
\hline$R E(\%)$ & 1.0251 & 1.0312 & 1.0233 & 1.0170 \\
\hline$R^{2}$ & 0.9997 & 0.9990 & 0.9997 & 0.9990 \\
\hline \multicolumn{5}{|l|}{ Validation } \\
\hline Number of iterations & 7 & 6 & 4 & 3 \\
\hline LOF (\%) (PCA) & 0.4702 & 0.7071 & 0.4697 & 0.6945 \\
\hline LOF (\%) (exp.) & 0.8215 & 0.9766 & 0.8213 & 0.9676 \\
\hline Variance (\%) & 99.9933 & 99.9905 & 99.9933 & 99.9906 \\
\hline $\operatorname{RMSEP}\left(\mu \mathrm{g} \mathrm{mL}^{-1}\right)$ & 0.4553 & 0.2065 & 0.4507 & 0.1809 \\
\hline$R E(\%)$ & 1.2335 & 1.1800 & 1.2210 & 1.0335 \\
\hline$R^{2}$ & 0.9987 & 0.9998 & 0.9987 & 0.9998 \\
\hline
\end{tabular}

CC are represented in Fig. 3. The spectral profile values were tested for each component by calculating their correlation with the pure spectrum of the drugs and excellent results were obtained: 0.9933 and 0.9983 for DOX and PYR, resp.
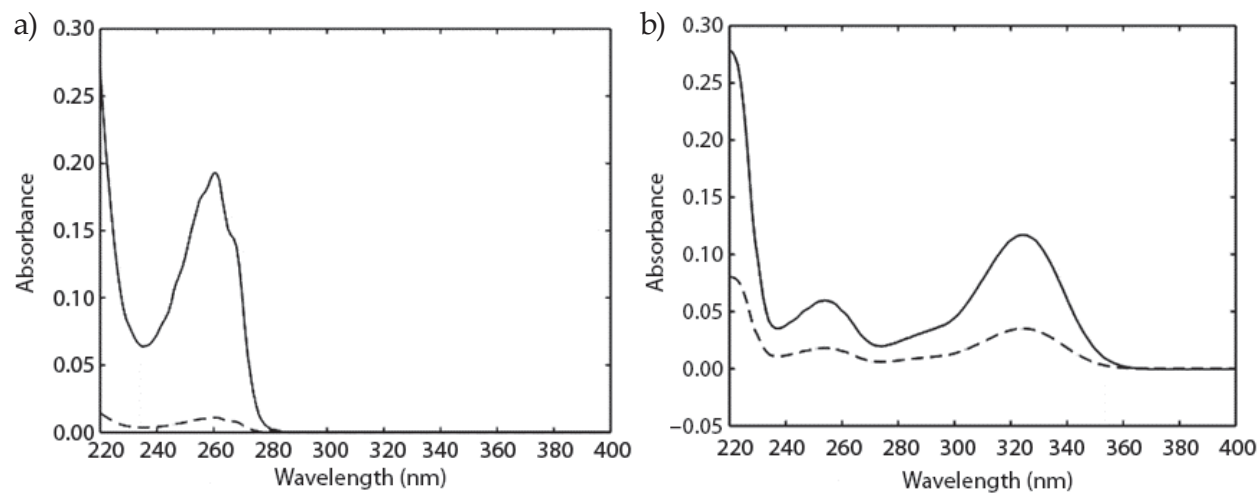

Fig. 3. Spectral profiles of the two analytes - normalized true (-) and estimated using MCR-ALS (--): a) DOX $\left(55 \mu \mathrm{g} \mathrm{mL}{ }^{-1}\right)$, b) PYR $\left(10 \mu \mathrm{g} \mathrm{mL}{ }^{-1}\right)$. 
P. Katsarov et al:: E Advanced spectrophotometric chemometric methods for resolving the binary mixture of doxylamine succinate and pyridoxine hydrochloride, Acta Pharm. 68 (2018) 61-73.

Table IV. Results of the validation test set prediction using PLS1, PLS2, RPM and MCR-ALS-CC methods

\begin{tabular}{|c|c|c|c|c|c|}
\hline \multicolumn{2}{|c|}{ Mixture } & \multicolumn{2}{|c|}{ PLS1 } & \multicolumn{2}{|c|}{ PLS2 } \\
\hline DOX & PYR & DOX & PYR & DOX & PYR \\
\hline \multicolumn{2}{|c|}{ True $\left(\mu \mathrm{g} \mathrm{mL}^{-1}\right)$} & \multicolumn{2}{|c|}{ Recovery $(\%)$} & \multicolumn{2}{|c|}{ Recovery (\%) } \\
\hline 30.0 & 10.0 & 100.66 & 98.73 & 100.54 & 99.30 \\
\hline 40.0 & 10.0 & 101.50 & 100.29 & 101.15 & 100.86 \\
\hline 30.0 & 15.0 & 101.33 & 99.84 & 100.86 & 100.44 \\
\hline 30.0 & 20.0 & 99.33 & 100.90 & 99.44 & 100.61 \\
\hline 30.0 & 30.0 & 99.33 & 100.19 & 99.19 & 100.01 \\
\hline 40.0 & 15.0 & 101.50 & 100.04 & 101.21 & 100.71 \\
\hline 40.0 & 20.0 & 99.25 & 100.09 & 99.40 & 99.94 \\
\hline 50.0 & 10.0 & 101.40 & 100.99 & 101.07 & 101.6 \\
\hline \multicolumn{2}{|c|}{ Mean (\%) } & 100.54 & 100.13 & 100.36 & 100.44 \\
\hline \multicolumn{2}{|c|}{ SD } & 1.06 & 0.70 & 0.87 & 0.71 \\
\hline \multicolumn{2}{|c|}{ Mixtures } & \multicolumn{2}{|c|}{ RPM } & \multicolumn{2}{|c|}{ MCR-ALS-CC } \\
\hline DOX & PYR & DOX & PYR & DOX & PYR \\
\hline \multicolumn{2}{|c|}{ True $\left(\mu \mathrm{g} \mathrm{mL}^{-1}\right)$} & \multicolumn{2}{|c|}{ Recovery $(\%)$} & \multicolumn{2}{|c|}{ Recovery (\%) } \\
\hline 30.0 & 10.0 & 100.45 & 99.20 & 100.23 & 99.53 \\
\hline 40.0 & 10.0 & 101.15 & 100.64 & 101.17 & 102.32 \\
\hline 30.0 & 15.0 & 101.12 & 100.30 & 101.72 & 101.44 \\
\hline 30.0 & 20.0 & 98.83 & 100.71 & 99.22 & 100.13 \\
\hline 30.0 & 30.0 & 98.53 & 100.05 & 99.43 & 100.31 \\
\hline 40.0 & 15.0 & 101.22 & 100.57 & 101.76 & 101.41 \\
\hline 40.0 & 20.0 & 98.77 & 99.99 & 98.94 & 99.69 \\
\hline 50.10 & 10.0 & 101.04 & 101.46 & 101.22 & 103.18 \\
\hline \multicolumn{2}{|c|}{ Mean (\%) } & 100.14 & 100.37 & 100.46 & 101.00 \\
\hline \multicolumn{2}{|c|}{ SD } & 1.21 & 0.66 & 1.15 & 1.31 \\
\hline
\end{tabular}

The calculated statistical parameters of calibration and validation for MCR-ALS and MCR-ALS-CC are listed in Table III.

As shown, the values of $L O F$ and percent of variance were comparable when both constraints (equality and correlation) were applied. On the other hand, convergence was achieved by different numbers of iterations. Applying the correlation constraint reduced the number of iterations for DOX and PYR. All parameters (RMSEC, RMSEP and RE) for both drugs indicated that correlation was more convenient than the equality constraint for final prediction, making MCR-ALS-CC the better model. The obtained results indicated that the prediction ability of MCR-ALS methods was better for PYR compared to DOX, which was also observed with the PLS models. In general, the analytical and statistical parameters 
P. Katsarov et al:: E Advanced spectrophotometric chemometric methods for resolving the binary mixture of doxylamine succinate and pyridoxine hydrochloride, Acta Pharm. 68 (2018) 61-73.

Table V. Recovery obtained by PLS1, PLS2, RPM and MCR-ALS-CC methods for assaying Cariban ${ }^{\circledR}$ capsules

\begin{tabular}{lcccccccc}
\hline \multirow{2}{*}{ Parameter } & \multicolumn{2}{c}{ PLS1 } & \multicolumn{2}{c}{ PLS2 } & \multicolumn{2}{c}{ RPM } & \multicolumn{2}{c}{ MCR-ALS-CC } \\
\cline { 2 - 9 } & DOX & PYR & DOX & PYR & DOX & PYR & DOX & PYR \\
\hline Recovery (\%) & 98.43 & 101.76 & 100.08 & 102.34 & 98.55 & 101.45 & 100.79 & 99.18 \\
SD & 1.44 & 1.38 & 1.37 & 1.19 & 1.29 & 1.08 & 0.72 & 0.69 \\
\hline
\end{tabular}

provided in Tables II and III confirmed that DOX could be analysed by both methods (PLS and MCR-ALS); however, PLS methods were more effective for PYR prediction.

Among the studied methods, PLS and PRM showed the best predictive ability for the analysis of DOX and PYR binary mixtures. In spite of that, the developed MCR-ALS methods had the advantage of predicting pure spectral profiles of the drugs and also the influence of interfering components in dosage forms.

After parameter optimization and the calibration step, the models were successfully applied to the estimation of DOX and PYR in the validation set. The mean recovery and standard deviation values are summarized in Table IV. The obtained results indicated a high predictive ability of all developed methods for drug quantification in the validation set.

\section{Analysis of commercial dosage forms}

The proposed PLS and MCR-ALS-CC methods were applied to simultaneous determination of DOX and PYR in commercial Cariban ${ }^{\circledR}$ capsules. The obtained statistical parameters are given in Table $\mathrm{V}$.

All methods proved to be reasonable analytical procedures to quantify both DOX and PYR in pharmaceutical formulations, with excellent recoveries of 98.43-102.34 \%. Better performance of MCR-ALS for drug quantification in dosage forms was probably due to the ability of the method to handle the negative contribution of the un-calibrated excipients in the formulation, which were not included in calibration samples.

\section{CONCLUSIONS}

The adopted multivariate methods showed similar performance for doxylamine and pyridoxine determination in binary mixtures. The predictive ability of PLS1 was comparable to that of PLS2 and PRM. However, a robust PRM algorithm demonstrated superiority over the other two PLS versions due to the initial detection and removal of outliers before calibration. MCR-ALS-CC gave the best output for drug quantification in dosage forms. All of the developed calibration procedures proved to be reliable methods for analyses of doxylamine and pyridoxine in pharmaceutical formulations.

Acronyms, abbreviations, symbols. - CCD - central composite design, CLS - classical least squares, DOX - doxylamine succinate, FDCs - fixed-dose combination drug products, FOM - figures of merit, HPTLC - high-performance thin-layer chromatography, ILS - inverse least squares, LOD - limit of detection, LOF - lack of fit, LOQ - limit of quantification, LVs - latent variables, MCR-ALS - multi- 
variate curve resolution-alternating least squares, MCR-ALS-CC - multivariate curve resolution-alternating least squares with correlation constraint, PBS - phosphate-buffered saline, PCA - principal component analysis, PCR - principal components regression, PCs - principal components, PLS - partial least squares, PRM - partial robust M-regression, PYR - pyridoxine hydrochloride, $R^{2}-$ squared correlation coefficient, RE - relative error of prediction, RMSEC - root mean square errors of calibration, RMSEP - root mean square errors of prediction, RP-HPLC - reversed-phase high-performance liquid chromatography, SVD - singular value decomposition

Acknowledgements. - The authors gratefully acknowledge financial support from the Medical University Plovdiv through university projects SDP-04/2015 and BG05M2OP001-2.009-0025.

\section{REFERENCES}

1. S. R. Slaughter, R. Hearns-Stokes, T. van der Vlugt and H. V. Joffe, FDA approval of doxylaminepyridoxine therapy for use in pregnancy, New Engl. J. Med. 370 (2014) 1081-1083; https://doi. org/10.1056/NEJMp1316042

2. N. Nuangchamnong and J. Niebyl, Doxylamine succinate-pyridoxine hydrochloride (Diclegis) for the management of nausea and vomiting in pregnancy: an overview, Int. J. Womens Health 6 (2014) 401-409; https://doi.org/10.2147/IJWH.S46653

3. M. De Luca, F. Oliverio, G. Ioele and G. Ragno, Multivariate calibration techniques applied to derivative spectroscopy data for the analysis of pharmaceutical mixtures, Chemometr. Intell. Lab. Syst. 96 (2009) 14-21; https://doi.org/10.1016/j.chemolab.2008.10.009

4. Z. Rasouli and R. Ghavami, Simultaneous detection of calcium and magnesium in various samples by calmagite and chemometrics data processing, Spectrochim. Acta A Mol. Biomol. Spectrosc. 169 (2016) 72-81; https://doi.org/10.1016/j.saa.2016.06.027

5. T. Azzouz and R. Tauler, Application of multivariate curve resolution alternating least squares (MCR-ALS) to the quantitative analysis of pharmaceutical and agricultural samples, Talanta 74 (2008) 1201-1210; https://doi.org/10.1016/j.talanta.2007.08.024

6. H. Darwish, A. Bakheit and A. Abdelhameed, Simultaneous quantitative analysis of olmesartan, amlodipine and hydrochlorothiazide in their combined dosage form utilizing classical and alternating least squares based chemometric methods, Acta Pharm. 66 (2016) 83-95; https://doi. org/10.1515/acph-2016-0004

7. K. S. Booksh and B. R. Kowalski, Theory of analytical chemistry, Anal. Chem. 66 (1994) 782A-791A; https://doi.org/10.1021/ac00087a001

8. European Pharmacopoeia, 8th ed., European Directorate for the Quality of Medicines \& Health Care, Strasbourg 2013, pp. 2112-2113, 3133-3134, 5602-5608.

9. P. Giriraj and T. Sivakkumar, Development and validation of a rapid chemometrics assisted RPHPLC with PDA detection method for the simultaneous estimation of pyridoxine $\mathrm{HCl}$ and doxylamine succinate in bulk and pharmaceutical dosage form, Chromatogr. Res. Int. 2014 (2014) 1-8; https://doi.org/10.1155/2014/827895

10. A. P. Argekar and J. G. Sawant, Simultaneous determination of pyridoxine hydrochloride and doxylamine succinate in tablets by HPTLC, J. Liq. Chromatogr. Relat. Technol. 22 (1999) 2051-2060; https://doi.org/10.1081/JLC-100101785

11. A. Pathak and S. J. Rajput, Simultaneous determination of a ternary mixture of doxylamine succinate, pyridoxine hydrochloride, and folic acid by the ratio spectra zero-crossing, double divisor ratio spectra derivative, and column high-performance liquid chromatographic methods, J. AOAC Int. 91 (2008) 1059-1069; https://www.ncbi.nlm.nih.gov/pubmed/18980119

12. S. C. Nayak, P. V. Kulkarni, V. Bhaskar and V. Chavhan, Development and validation of UV spectrophotometric method for simultaneous estimation of doxylamine succinate and pyridoxine hy- 
drochloride in bulk and tablet dosage forms, Int. J. Pharm. Pharm. Sci. 5 (2013) 390-393; http://www. ijppsjournal.com/Vol5Suppl3/7343.pdf

13. K. S. Nataraj, Y. Suvarna, and G. Venkateswari, Development and validation of method for simultaneous estimation of pyridoxine hydrochloride and doxylamine succinate in tablet dosage form by first order derivative spectroscopy, Int. J. Pharm. Pharm. Sci. 5 (2013) 388-390; http://www.ijppsjournal.com/Vol5Issue1/6289.pdf

14. P. Bhamre, A. Pathak and S. Rajput, Simultaneous determination of doxylamine succinate, pyridoxine hydrochloride and folic acid by chemometric spectrophotometry, Int. J. Pharm. Biol. Sci. 4 (2013) 738-749; http://www.ijpbs.net/download.php?download_file=vol-4/issue-1/pharma/72. pdf\&did $=2061$

15. C. A. Nunes, M. P. Freitas, A. C. M. Pinheiro and S. C. Bastos, Chemoface: a novel free user-friendly interface for chemometrics, J. Braz. Chem. Soc. 23 (2012) 2003-2010; http://www.scielo.br/scielo. php?script=sci_arttext\&pid=S0103-50532012001100007

16. S. Wold, M. Sjöström and L. Eriksson, PLS-regression: a basic tool of chemometrics, Chemometr. Intell. Lab. Syst. 58 (2001) 109-130; https://doi.org/10.1016/S0169-7439(01)00155-1

17. S. Serneels, C. Croux, P. Filzmoser and P. J. Van Espen, Partial robust M-regression, Chemometr. Intell. Lab. Syst. 79 (2005) 55-64; https://doi.org/10.1016/j.chemolab.2005.04.007

18. R. Tauler, A. Smilde and R. Kowalsky, Selectivity, local rank, three-way data analysis and ambiguity in multivariate curve resolution, J. Chemom. 9 (1995) 31-58; https://doi.org/10.1002/cem.1180090105

19. D. L. Massart, B. G. M. Vandeginste, L. M. C. Buydens, S. De Jong, P. J. Lewi and J. Smeyers-Verbeke, Handbook of Chemometrics and Qualimetrics: Part A, Elsevier, Amsterdam 1997.

20. R. Bro and S. De Jong, A fast non-negativity-constrained least squares algorithm, J. Chemom. 11 (1997) 393-401; https://doi.org/10.1002/(sici)1099-128x(199709/10)11:5\%3C393::aid-cem483\%3E3.0.co;2-1

21. A. de Juan, J. Jaumot and R. Tauler, Multivariate curve resolution (MCR). Solving the mixture analysis problem, Anal. Methods 6 (2014) 4964-4976; https://doi.org/10.1039/C4AY00571F

22. R. R. de Oliveira, K. M. G. de Lima, R. Tauler and A de Juan, Application of correlation constrained multivariate curve resolution alternating least-squares methods for determination of compounds of interest in biodiesel blends using NIR and UV-visible spectroscopic data, Talanta 125 (2014) 233-241; https://doi.org/10.1016/j.talanta.2014.02.073

23. A. C. Olivieri, H. C. Goicoechea and F. A. Inón, MVC1: an integrated MatLab toolbox for first-order multivariate calibration, Chemometr. Intell. Lab. Syst. 73 (2004) 189-197; https://doi.org/10.1016/j.chemolab.2004.03.004

24. M. Daszykowski, S. Serneels, K. Kaczmarek, P. Van Espen, C. Croux and B. Walczak, TOMCAT: A MATLAB toolbox for multivariate calibration techniques, Chemometr. Intell. Lab. Syst. 85 (2007) 269-277; https://doi.org/10.1016/j.chemolab.2006.03.006

25. J. Jaumot, A. de Juan and R. Tauler, MCR-ALS GUI 2.0: new features and applications, Chemometr. Intell. Lab. Syst. 140 (2015) 1-12; https://doi.org/10.1016/j.chemolab.2014.10.003

26. M. Ristila, J. M. Matxain, Å. Strid and L. A. Eriksson, pH-dependent electronic and spectroscopic properties of pyridoxine (vitamin B6), J. Phys. Chem. B 110 (2006) 16774-16780; https://doi.org/10.1021/ jp062800n

27. H. Goicoechea and A. Olivieri, Simultaneous determination of phenobarbital and phenytoin in tablet preparations by multivariate spectrophotometric calibration, Talanta 47 (1998) 103-108; https://doi.org/10.1016/S0039-9140(98)00070-8

28. V. Centner, O. E. de Noord and D. L. Massart, Detection of nonlinearity in multivariate calibration, Anal. Chim. Acta 376 (1998) 153-168; https://doi.org/10.1016/S0003-2670(98)00543-1

29. F. Allegrini and A. C. Olivieri, IUPAC-consistent approach to the limit of detection in partial leastsquares calibration, Anal. Chem. 86 (2014) 7858-7866; https://doi.org/10.1021/ac501786u 